\title{
Isochrone-cloud fitting of the extended main-sequence turn-off of young clusters ${ }^{\star}$
}

\author{
C. Johnston ${ }^{1}$, C. Aerts ${ }^{1,2,3}$, M. G. Pedersen ${ }^{1}$, and N. Bastian ${ }^{4}$ \\ ${ }^{1}$ Instituut voor Sterrenkunde, KU Leuven, Celestijnenlaan 200D, 3001 Leuven, Belgium \\ e-mail: colecampbell.johnston@kuleuven. be \\ 2 Department of Astrophysics, IMAPP, Radboud University Nijmegen, PO Box 9010, 6500 GL Nijmegen, The Netherlands \\ 3 Max Planck Institute for Astronomy, Koenigstuhl 17, 69117 Heidelberg, Germany \\ 4 Astrophysics Research Institute, Liverpool John Moores University, 146 Brownlow Hill, Liverpool L3 5RF, UK
}

Received 22 August 2019 / Accepted 1 October 2019

\begin{abstract}
Context. Extended main-sequence turn-offs (eMSTOs) are a commonly observed property of young clusters. A global theoretical interpretation for eMSTOs is still lacking, but stellar rotation is considered a necessary ingredient to explain eMSTOs.

Aims. We aim to assess the importance of core-boundary and envelope mixing in stellar interiors for the interpretation of eMSTOs in terms of one coeval population.

Methods. We constructed isochrone-clouds based on interior mixing profiles of stars with a convective core calibrated from asteroseismology of isolated galactic field stars. We fitted these isochrone-clouds to the measured eMSTO to estimate the age and core mass of the stars in the two young clusters NGC 1850 and NGC 884, assuming one coeval population and by fixing the metallicity to the one measured from spectroscopy. We assessed the correlations between the interior mixing properties of the cluster members and their rotational and pulsational properties.

Results. We find that stellar models based on asteroseismically-calibrated interior mixing profiles lead to enhanced core masses of eMSTO stars. Additionally, these models can explain a significant fraction of the observed eMSTOs of the two considered clusters in terms of one coeval population of stars, which have similar ages to those in the literature, given the large uncertainties. The rotational and pulsational properties of the stars in NGC 884 are not sufficiently well known to perform asteroseismic modelling as it is achieved for field stars from space photometry. The stars in NGC 884 for which we have $v \sin i$ and a few pulsation frequencies show no correlation between these properties and the core masses of the stars that set the cluster age.

Conclusions. Future cluster space asteroseismology may allow for the interpretation of the core masses in terms of the physical processes that cause them, based on the modelling of the interior mixing profiles for the individual member stars with suitable identified modes.
\end{abstract}

Key words. asteroseismology - stars: interiors - stars: oscillations - stars: rotation - galaxies: star clusters: general

\section{Introduction}

The theoretical understanding of the physical processes in stellar interiors and their implementation in modern stellar structure and evolution (SSE) codes are fundamental cornerstones of astrophysics. To this end, stellar astrophysics has benefited tremendously from the exponential development of asteroseismology, which is the investigation of stellar interiors through the characterisation and modelling of stellar oscillation modes. Major progress has been achieved in the past decade thanks to space-based photometric missions, such as CoRoT, Kepler, BRITE, K2, and TESS.

One of the great benefits of these long time-base, high dutycycle high-precision observations has been the probing of the deep stellar interior of stars born with a convective core. This can be achieved through asteroseismology of long-period (order of days) gravity-mode oscillations ( $g$-modes hereafter), which is impossible for the ground-based data of stars in the corehydrogen burning phase. The $g$-modes in intermediate-mass

\footnotetext{
* Isochrone-cloud files are only available at the CDS via anonymous ftp to cdsarc.u-strasbg.fr $(130.79 .128 .5)$ or via http: //cdsarc.u-strasbg.fr/viz-bin/cat/J/A+A/632/A74
}

stars with $M_{\star} \in[1.3,8.5] M_{\odot}$ propagate between the stellar surface and the convective core. Such resonant $g$-modes are triggered by a flux-blocking mechanism at the bottom of the convective envelope for the F-type $\gamma$ Doradus stars (Dupret et al. 2005; Bouabid et al. 2013) and by a heat $(\kappa)$ mechanism in the outer radiative envelope acting upon the partial ionisation layers or iron-like elements for slowly pulsating B (SPB) stars (Miglio et al. 2007; Szewczuk \& Daszyńska-Daszkiewicz 2017). Gravity-mode asteroseismology requires uninterrupted monitoring of tiny brightness variations at $\mu$ mag level during several months and was first achieved for five-month long CoRoT light curves (Degroote et al. 2010; Pápics et al. 2012).

The amount of mass in the convective core drives the evolution of intermediate and high-mass stars and determines their age at the end of a given nuclear core-burning phase. It is therefore of utmost importance to estimate the amount of mixing that occurs in the transition layers between the convective core and the bottom of the radiative envelope. Gravitymodes allow one to probe this particularly important region. The exploitation of detected $g$-mode oscillations reveals that stars rotate nearly rigidly throughout the main-sequence (MS, Kurtz et al. 2014; Saio et al. 2015; Murphy et al. 2016; Aerts et al. 2017; Van Reeth et al. 2018; Li et al. 2019a). This points to 
an efficient angular momentum (AM) transport mechanism that is currently not included in standard SSE models. Such efficient AM transport and near-rigid rotation not only occur during the MS but also during core-helium burning, that is, in phases when stars have a convective core (e.g. Aerts et al. 2019, for a review).

Both heat-driven resonant $g$-modes, which excited by the $\kappa$ mechanism, and damped travelling internal gravity waves (IGWs) that are triggered stochastically at the interface of the convective core and the radiative envelope have been suggested as missing ingredients to remedy this discrepancy in the theory of AM transport (Rogers et al. 2013; Aerts 2015; Rogers 2015; Townsend et al. 2018). While IGWs which are excited by the convective core do not depend on metallicity, their propagation and dissipation properties are poorly understood. Recent CoRoT, $\mathrm{K} 2$, and TESS data have revealed detection of IGWs in almost all OB stars, both in the Milky Way and in the Large Magellanic Cloud (Bowman et al. 2019a,b). Hence, demonstrating that low metallicity stars, which do not undergo resonant heat-driven $g$-modes, can undergo efficient angular momentum transport via IGWs.

High-precision ageing, via asteroseismic estimation of the convective core mass, only became possible due to the fouryear long light curves assembled with the Kepler mission, which allowed for the identification of the degree of detected $g$-mode frequencies from their period spacing patterns. The stellar age can be estimated from such data by assessing the core mass of the star. This is done from measuring the level of near-core boundary mixing (CBM), in the form of convective core overshooting, in single and binary B-type stars (e.g. Moravveji et al. 2015, 2016; Kallinger et al. 2017; Szewczuk \& DaszyńskaDaszkiewicz 2018; Johnston et al. 2019a) and in single F-type stars, for example (e.g. Van Reeth et al. 2016; Mombarg et al. 2019). The asteroseismically calibrated levels of core overshooting led to values between typically 0.1 and 0.5 times the local pressure scale height, resulting in increased core masses compared to standard SSE models without extra mixing at the nearcore boundary. With the exception of one magnetic $g$-mode pulsator (Buysschaert et al. 2018), higher-than-standard core masses were found for almost all studied $g$-mode pulsators of intermediate mass (some 50 stars), covering the entire corehydrogen burning phase, irrespective of their level of rotation.

The assumption that the core masses of intermediate-mass stars are solely influenced by overshooting is a simplification. Indeed, in reality convective penetration by plumes, overshooting, rotation, and oscillatory motions due to resonant modes and IGWs all interplay and imply the occurrence of an overall net near-CBM profile. This overall CBM may further be affected (or not) by core magnetism (Fuller et al. 2019) or by tides in the case of a close binary (Song et al. 2013). Moreover, chemical mixing also occurs in the radiatively stratified envelope. Such envelope mixing (hereafter denoted as REM) may be of either microscopic or macroscopic nature. Inherently, these two forms of mixing produce opposite effects in a given layer of the radiative envelope. Macroscopic mixing as triggered by rotation, (semi)convection, convective envelope boundary mixing (via entrainment, penetration, or overshooting, see Viallet et al. 2015), waves, magnetism, tides, etc, occurs on relatively short timescales, and homogeneously mixes the layer. Alternatively, mixing of microscopic origin due to atomic diffusion occurs on relatively long timescales. Here, we consider atomic diffusion as the combined effect of gravitational settling, thermal diffusion, concentration diffusion, and radiative levitation (Thoul et al. 1994; Michaud et al. 2015; Dotter et al. 2017). These processes can alter chemical gradients and create relative overor under-abundances of given elements within the stably stratified radiative region. The timescales involved in these processes determine which effect is dominant. If any large scale macroscopic mixing mechanism is active on a significantly shorter timescale than atomic diffusion, the layer will be homogeneously mixed, effectively erasing the fingerprint of atomic diffusion. We assume this to be the case in our models, such that the REM is only of macroscopic origin. Unfortunately, the overall profile of neither the CBM nor the REM is well understood, as it cannot be derived from first principles (e.g. Salaris \& Cassisi 2017, for a review). Recently, however, asteroseismic modelling of intermediate-mass stars has been able to probe the level of this $\mathrm{CMB}+\mathrm{REM}$ mixing profile from $g$-mode periodspacing patterns and their deviations from uniformity (e.g. Moravveji et al. 2015, 2016; Schmid \& Aerts 2016; Mombarg et al. 2019). Indeed, $g$-mode asteroseismology has been proven to be sensitive to the morphology of the CBM+REM mixing profile within stars (Pedersen et al. 2018), as well as to the temperature gradient within the CBM region adjacent to the convective core (Michielsen et al. 2019).

Aside from $g$-mode asteroseismology, numerous studies which performed isochrone modelling of binary stars have also indicated the need for enhanced core masses (e.g. Ribas et al. 2000; Claret 2007; Tkachenko et al. 2014; Higl \& Weiss 2017; Claret \& Torres 2019; Johnston et al. 2019b, among others). However, binary modelling delivers less strong probing than $g$-modes and uncertainties remain in the calibration of CBM using only binary constraints (Constantino \& Baraffe 2018; Johnston et al. 2019a). Johnston et al. (2019a) combined binary and $g$-mode asteroseismic modelling to show that derived core mass estimates are in that case more constrained than CBM estimates from binarity alone for a few double-lined $g$-mode pulsating binaries observed by Kepler. Johnston et al. (2019b) made the same assertion for eclipsing binaries, stressing that the core mass should be estimated and calibrated, rather than the efficiency of a single mixing mechanism.

The asteroseismic modelling of individual single and binary field stars discussed above has shown that mixing with a whole variety of levels and profiles is active in intermediate-mass stars. The major consequence of these asteroseismically derived CBM and REM levels for stellar evolution is an increase in the core mass of the star. As a result, asteroseismology has provided a range of stellar core mass fractions along the MS, because CBM brings fresh fuel into the core (for a summary of CBM and REM values, see the analyses by Briquet et al. 2007; Moravveji et al. 2015, 2016; Schmid \& Aerts 2016; Buysschaert et al. 2018; Hendriks \& Aerts 2019; Mombarg et al. 2019; Johnston et al. 2019a, for B- and F-type stars, respectively, hereafter jointly referred to as MfA: "Mixing-from-Asteroseismology"). One of the most important implications of stars having a plethora of mixing profiles and enhanced core masses is that the terminalage MS (TAMS) is not a single line in the Hertzsprung-Russel diagram (HRD), but rather an entire region. This has so far largely been ignored in cluster studies.

Extended main-sequence turn-offs (eMSTOs) are ubiquitous in colour-magnitude diagrams (CMDs) of young clusters in the Milky Way and in the Large and Small Magellanic Clouds (LMC and SMC, e.g. Mackey et al. 2008; Milone et al. 2018; Marino et al. 2018a; Cordoni et al. 2018). The eMSTO is characterised by an apparently broad TAMS region in the CMD, covering a given width in colour, depending on the particular cluster. Since its discovery, the mechanism behind the eMSTO has been a matter of intense debate. Several mechanisms have 
been proposed to explain the eMSTO phenomenon, such as age spreads, binaries and binary interaction products, near-critical rotation, and convective penetration, with no single mechanism being able to explain the full breadth of observed morphologies (e.g. Li et al. 2019b; Gossage et al. 2018, and references therein).

The observed eMSTOs have been associated with fast surface rotation as revealed by spectroscopy (e.g. Dupree et al. 2017; Kamann et al. 2018; Marino et al. 2018b; Bastian et al. 2018). While some clusters with high-mass stars at the eMSTO show a correlation between $\mathrm{H} \alpha$ emission and location in the CMD (Bodensteiner et al. 2019), others such as the double cluster $h$ and $\chi$ Persei reveal no relation between colour and rotational velocities ( $\mathrm{Li}$ et al. 2019b). Furthermore, in independent studies, both Li et al. (2019b) and Beasor et al. (2019) demonstrate that models consisting of a single population of stars with the same age and metallicity but with a variety of rotation rates cannot explain the full morphology of this double cluster and suggest that other mechanisms such as binary interaction could be important. While in general stellar evolution models including rotational effects and magnetic braking offer improved isochrone fits to eMSTOs in terms of coeval populations compared to non-rotating non-magnetic models (Georgy et al. 2019), they fail to explain the full morphology of the eMSTO in clusters of young to intermediate ages (e.g. Milone et al. 2018; Goudfrooij et al. 2018; Li et al. 2019b). Whether this is caused by limitations in the input physics used in the models, or the fitting methodology is yet to be determined. Goudfrooij et al. (2018) pointed out that the MS of young clusters are narrow for stellar masses below $\sim 1.4 M_{\odot}$ and broaden for all stars above this "cut off" mass. This minimal eMSTO mass coincides with the mass for which both a well developed convective core and non-radial $g$-modes occur in MS stars (e.g. Van Reeth et al. 2015; Pápics et al. 2017). As such, we investigate if the asteroseismically calibrated mixing mechanisms discussed above, and particularly their consequence in terms of increased core mass, play a role in the formation of the eMSTO of young open clusters.

Yang \& Tian (2017) already considered the case of allowing for different levels of convective penetration as the mechanism for CBM for non-rotating stellar models of a coeval population and showed that this can explain the eMSTO to some extent. We generalise this approach to investigate to what level the mixing profiles calibrated by asteroseismology of stars with a convective core can explain the eMSTOs of young clusters. We do this by constructing so-called isochrone-clouds first introduced by Johnston et al. (2019a). These are areas in the HRD covered by a coeval population of stars having various masses and fractional core masses due to different interior mixing profiles. These areas naturally form an extended TAMS region. Unlike Yang \& Tian (2017), who only considered penetrative convection with un-calibrated large values of the penetration distance up to 0.7 times the local pressure scale height, we consider the general case of both CBM and REM and limit the overall amount of CBM and REM to measured levels calibrated by asteroseismology of intermediate- and high-mass field stars as achieved by the MfA studies. We fitted isochrone-clouds to CMDs of two prototypical clusters with observed eMSTOs to illustrate the capacity and limitations of isochrone clouds in explaining this observed phenomenon. So far, pulsational wave mixing leading to larger core masses has been ignored in cluster studies. Here, we remedy this lack in the current exploratory paper.

\section{Interior mixing and the concept of isochrone-clouds}

\subsection{Interior mixing}

The interior mixing profile results from the transport of chemical elements inside the star. This profile cannot be determined from the basic equations of stellar structure. It is the net effect of several mechanisms (inter)acting together in 3D to produce an overall mixing profile that is then simplified into a 1D description (e.g. Arnett et al. 2015). Here, we are concerned with stars that have a convective core and we denote the mixing profile as a function of radial coordinate: $D_{\text {mix }}\left(r>r_{\mathrm{cc}}\right)$ with $r_{\mathrm{cc}}$ the boundary of the convective core of the star according to the Ledoux criterion of convection. As mentioned in Sect. 1, physical processes that contribute to the overall mixing profile are numerous.

Near-critical rotation and its geometrical consequences in the CMD due to the von Zeipel effect have been used to explain the eMSTO in young clusters. Evidence for such an interpretation has been corroborated by spectroscopically determined $v \sin i$ measurements of cluster members (e.g. Bastian et al. 2016; Dupree et al. 2017; Marino et al. 2018a,b). This has motivated eMSTO fitting by using stellar evolution models based on rotationally-induced mixing (e.g. Brott et al. 2011; Lagarde et al. 2012; Milone et al. 2018). Early versions of such models had been extensively studied to explain several observed phenomena in isolated field stars (e.g. Talon et al. 1997), binary systems (e.g. de Mink et al. 2009), and stellar populations (e.g. Chaboyer et al. 1995). Such models help improve the modelling of the eMSTO of the youngest clusters (e.g. Niederhofer et al. 2015). However, rotation alone cannot explain the morphology observed for clusters of intermediate age between a few hundred million years and a few giga-years (e.g. Goudfrooij et al. 2017). Those clusters older than a giga-year have turnoffs in the mass range $\sim 1.4 M_{\odot}$ (Goudfrooij et al. 2018) and can be well interpreted by adding the phenomenon of magnetic braking due to their convective envelope to rotational mixing Georgy et al. (2019).

Stars born with masses increasing from $\sim 1.3$ to $\sim 1.8 M_{\odot}$ not only lose their convective envelope but they also transfer from a radiative core to a well-mixed convective core, which contains between $\sim 10$ and $30 \%$ of the total mass, depending on the level and efficiency of CBM. Moreover, stars with a well-developed convective core are subject to a whole spectrum of dissipative IGWs, causing pulsational wave mixing throughout the radiative envelope of the star (Rogers \& McElwaine 2017). Space asteroseismology by MfA delivered calibrations for the profiles of CBM and REM, denoted here as $D_{\mathrm{CBM}}\left(r_{\mathrm{env}}>r>r_{\mathrm{cc}}\right)$ with $r_{\mathrm{env}}$ the position of the bottom of the fully radiative envelope, and $D_{\text {REM }}\left(r_{\text {env }}<r\right)$, whatever their (multiple) physical origins such as rotation, waves, tides, etc. We define $r_{\text {env }}$ to be the radial coordinate where $D_{\mathrm{REM}}=D_{\mathrm{CBM}}$, which is always uniquely defined in our case as our CBM is described by a constantly decreasing function, and our REM is described by a constantly increasing function. This location is dependent on both the amount of CBM and REM, as a small amount of CBM and large amount of REM will result in the smallest $r_{\text {env }}$, and conversely a large amount of CBM and a small amount of REM will result in the largest $r_{\text {env }}$. As such, we determine the extent of the CBM region as $\mathrm{d}_{\mathrm{CBM}}=r_{\mathrm{env}}-r_{\mathrm{cc}}$. Space asteroseismology did not reveal any correlation between the rotation frequency and the level of CBM or REM (Mombarg et al. 2019). This lack of correlation between rotation and mixing is in line with the early findings by Aerts et al. (2014) based on ground-based asteroseismology and the nitrogen surface abundance of a sample of OB-type field stars. 
Here, we investigate if and to what extent the levels of CBM and REM, and their resulting enhanced core-masses, as found from asteroseismology are capable in explaining the width and the morphology of the eMSTOs observed for young clusters.

\subsection{Stellar structure and evolution models}

In this work, we make use of the MESA stellar structure and evolution code (Paxton et al. 2011, 2013, 2015, 2018, 2019). As many such codes, MESA solves the equations of stellar structure in a diffusive approach. To this end, regions that are unstable against convection have a high diffusive mixing coefficient such that the material can be considered as instantaneously and fully mixed. The chemical mixing in radiative layers caused by each considered mechanism is implemented with its own mixing coefficient. The overall mixing profile in such layers is then the sum of the individual mixing phenomena in the regions inside the star wherever that mechanism is active. We point out that MESA's diffusive approach is different from codes that adopt an advective numerical scheme for the treatment of rotation and its consequences for mixing and angular momentum transport (such as the Geneva/STAREVOL or CESTAM codes, cf. Ekström et al. 2012; Marques et al. 2013, respectively). Detailed asteroseismic comparisons between advective and diffusive treatments of element transport in stellar models have yet to be carried out.

We calculate non-rotating spherically symmetric equilibrium models with MESA (version r-10108) and take a pragmatic approach by considering one global free parameter for the level of mixing for each of two areas inside the star: (1) CBM and (2) REM. In our scheme, CBM encompasses all forms of mixing active in the near core regions and is approximated by the implementation of diffusive exponential overshooting with a single free parameter, $f_{\text {ov }}$, given by:

$D_{\mathrm{ov}}=D_{0, \mathrm{cc}} \exp \frac{-2\left(r-r_{0}\right)}{f_{\mathrm{ov}} H_{p, \mathrm{cc}}}$,

where $r_{0}$ is the location at which the exponential function begins, $D_{0}$ is the diffusive mixing coefficient at $r_{0}$, and $H_{p, \text { cc }}$ is the local pressure scale height at the boundary of the convective core (Freytag et al. 1996; Paxton et al. 2011). The free parameter $f_{\mathrm{ov}}$ is then a scaling factor which determines how rapidly the exponential mixing profile decays. For the REM, we rely on a mixing profile due to IGW that scales with the inverse square-root of the density profile, as calibrated by simulations (Rogers \& McElwaine 2017) and $g$-mode asteroseismology (Pedersen et al. 2018). This REM profile has an efficiency level set at the outward end where the CBM region meets the bottom of the radiative envelope, requiring only one free parameter. A schematic representation of these two mixing profiles outside the convective core can be seen in the left panel of Fig. 1, for a model of $3 M_{\odot}$ with central hydrogen fraction $X_{\mathrm{c}}=0.3$ for the two combinations $\left(D_{\mathrm{CBM}}, D_{\mathrm{REM}}\right)=(0.005,1)$ and $(0.040,100)$ expressed in local pressure scale height and $\mathrm{cm}^{2} \mathrm{~s}^{-1}$ for $D_{\mathrm{CBM}}$ and $D_{\mathrm{REM}}$, respectively. This implies that we consider a global mixing profile $D_{\text {mix }}(r)$ described by two free parameters, whose observed ranges are calibrated from asteroseismology, beyond the conventional convective core boundary (hence for $r>r_{\mathrm{cc}}$ ), where $r_{\mathrm{cc}}$ is set by the Ledoux criterion. Thus we assume that the rotation of the cluster stars is sufficiently slow to ignore geometrical deformation due to the centrifugal force, which allows us to consider 1D spherically symmetric evolution models. Following Gagnier et al. (2019), our methodology is appropriate for stars rotating up to about $\sim 50 \%$ of their critical Keplerian rate at birth.

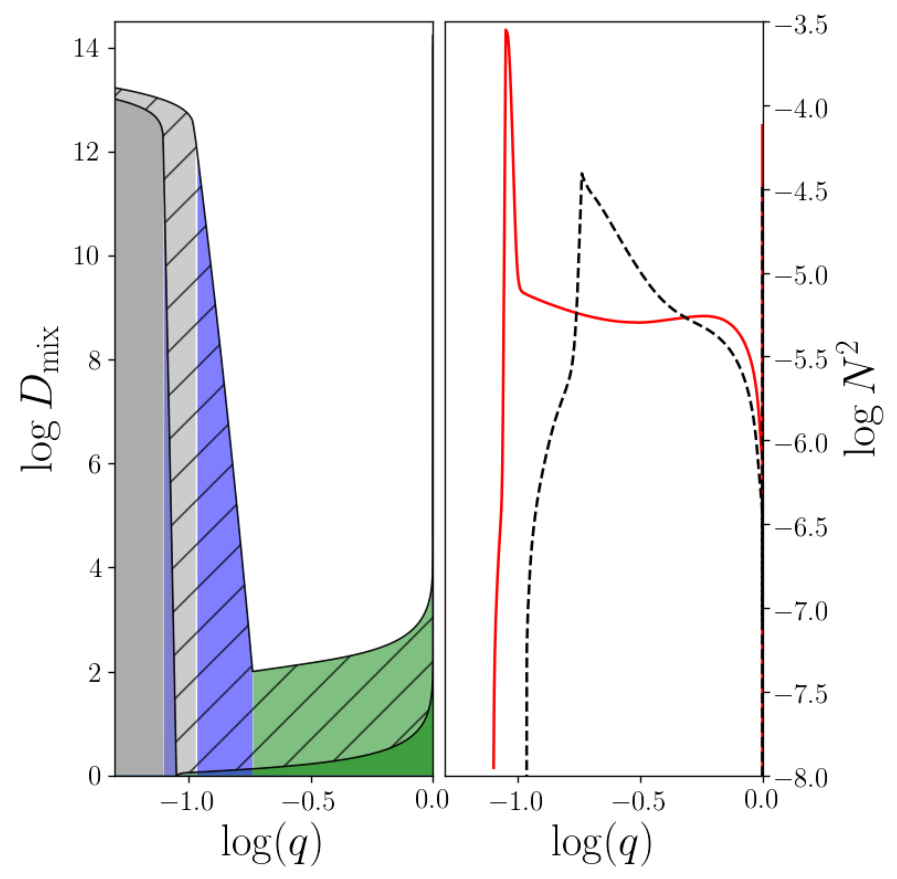

Fig. 1. Comparative plots of stellar model interiors. Left: mixing profiles for two stellar models of $3 M_{\odot}$ and $X_{\mathrm{c}}=0.3$ for the convective core (grey), convective boundary region (CBM, blue) and radiative envelope region (REM, green) plotted against the logarithm of the mass coordinate $q=m / M_{\star}$. The model with mixing profiles indicated as hatched regions has eight times higher CBM and 100 times higher REM than the other model, leading to an increase in core mass of $36.5 \%$. Right: logarithm of $N^{2}$ of the two models plotted against the logarithm of the mass coordinate $q$. The solid red line denotes $N^{2}$ of the model with a minimum amount of interior mixing, while the dashed black line denotes $N^{2}$ of the model with eight times higher CBM and 100 times higher REM.

Aside from abundance changes, the most pronounced effect of $\mathrm{CBM}+\mathrm{REM}$ is the increase in convective core mass throughout the MS phase. In fact, the MfA studies have shown that the $g$-modes are able to assess the mass in the convective core at a certain age. The difference in core mass and $N(r)$ for the two example models shown schematically in the left panel of Fig. 1 is apparent. As can be seen, the star with less mixing has both a less massive core (grey) and CBM region (blue) compared to the star with more mixing (hatched). The right panel compares the Brunt-Vaïsälä (BV) frequency (also termed buoyancy frequency) in the near-core region of these two stars (the model with minimum interior mixing denoted by the solid red line, the model with enhanced mixing denoted by the dashed black line). The BV frequency, $N(r)$, is a natural frequency that occurs inside a star set by the local temperature gradients and the $\mu$-gradient, with $\mu$ the mean molecular weight. It can be approximated as

$N^{2}=\frac{g}{H_{\mathrm{P}}}\left[\delta\left(\nabla_{\mathrm{ad}}-\nabla\right)+\varphi \nabla_{\mu}\right]$,

with

$\nabla=\frac{\partial \ln T}{\partial \ln P}, \nabla_{\mathrm{ad}}=\left(\frac{\partial \ln T}{\partial \ln P}\right)_{\mathrm{S}}, \nabla_{\mu}=\frac{\partial \ln \mu}{\partial \ln P}$

and

$\delta=\left(\frac{\partial \ln \rho}{\partial \ln T}\right)_{\mathrm{P}, \mu}, \varphi=\left(\frac{\partial \ln \rho}{\partial \ln \mu}\right)_{\mathrm{P}, \mathrm{T}}$,

where $\rho$ is the density, $P$ the pressure, $T$ the temperature, and $S$ the entropy. Both the interior mixing properties and the shrinking 

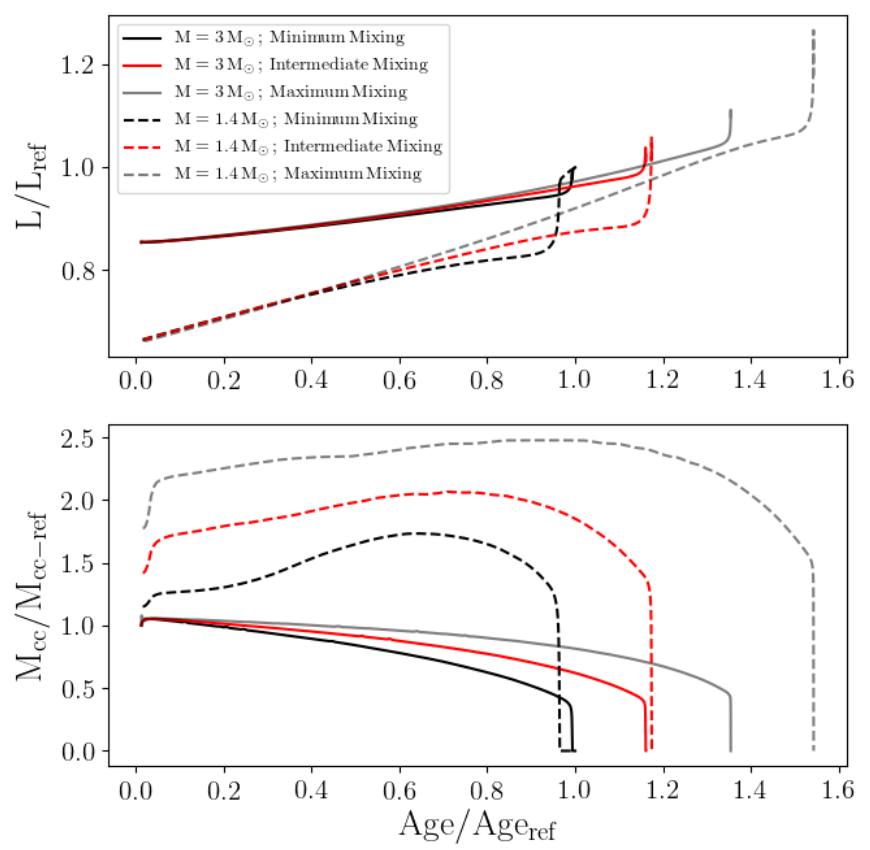

Fig. 2. Comparitive plots of stellar quantities over time. Top: luminosity of evolutionary models with varied amounts of mixing with reference to the luminosity and age of the model with the minimum amount of mixing at the TAMS. Bottom: convective core mass of evolutionary models with varied amounts of mixing with reference to the age of the model with the minimum amount of mixing at the TAMS and the core mass of the same model at the ZAMS.

convective core cause a $\mu$-gradient in the zone left behind, affect the local behaviour of $N(r)$. This local behaviour sets the propagation cavity of $g$-modes (see Aerts et al. 2019, for a through discussion of the probing power of such modes). The difference in core mass due to CBM+REM reveals itself in a deviating position along the evolutionary track in the CMD compared to the position of stars with $M>1.4 M_{\odot}$ that experience no mixing beyond the convective core boundary, while the difference in $N(r)$ affects the properties of the gravity modes. This the reason why asteroseismology can lead to a high-precision estimate of the core mass for a star with detected and identified resonant $g$-modes, as demonstrated by MfA.

At a given age, enhanced mixing produces a more massive core with respect to a star with lower levels of mixing at that age, which on the HRD in effect mimics the evolutionary track of an initially more massive star. In other words, the increased core mass results in an increased luminosity, radius, and temperature with respect to the case of no mixing beyond the convective core boundary. To illustrate the effects of increased mixing, in Fig. 2 we compare evolutionary tracks of a $1.4 M_{\odot}$ and a $3 M_{\odot}$ star, each with three levels of mixing: (1) minimum amount of mixing, (2) an intermediate amount of mixing (factor four enhancement in CBM and factor ten enhancement in REM), and (3) a maximum amount of mixing (factor eight enhancement in CBM and factor 100 enhancement in REM). The age is normalised with reference to the age at the TAMS of case 1, with a minimum amount of mixing. Likewise, the luminosity is normalised with reference to the luminosity at the TAMS of case 1. At the TAMS, the convective core mass is zero so we normalise the convective core mass with reference to the convective core mass of case 1 at the zero-age main-sequence (ZAMS). From Fig. 2 it is seen that enhanced CBM will cause deviations in core mass and luminosity from those cases where no CBM mixing is considered.

\subsection{Isochrone-clouds}

The concept of isochrone-clouds has been introduced by Johnston et al. (2019a,b) in order to bridge asteroseismically calibrated $D_{\text {mix }}(r)$ profiles and isochrone fitting of pulsating and eclipsing binaries, respectively. We briefly discuss their construction below.

Traditionally, an isochrone is constructed by interpolating in a grid of evolutionary tracks, all computed with the same fixed input physics yet described by a vector of free parameters $\theta$, to a desired age, $\tau$. The vector $\theta$ minimally consists of the birth mass and the initial chemical composition $(X, Z)$ with $X$ the initial hydrogen mass fraction and $Z$ the metallicity. Additionally, for more dimensional model grids, $\theta$ may also contain, for example, the mixing length parameter of convection, $\alpha_{\text {mlt }}$, the core overshoot parameter(s), etc. Thus, a given isochrone is described by the vector $\phi=\left\langle\theta_{i}, \tau_{j}\right\rangle$. For the construction of our isochroneclouds, we followed the isochrone construction as defined by Dotter (2016), to which we refer for details.

In this work, we fix $X=0.71$ and adopt the solar chemical mixture as in Asplund et al. (2009) while relying on the OP opacity tables (Seaton 2005). The vector $\theta_{i}$ corresponds to choices for the input parameters $\left(M, Z, D_{\mathrm{CBM}}, D_{\mathrm{REM}}\right)$, the latter two components being the free parameters that go into the computation of the overall mixing profile $D_{\text {mix }, i}(r)$ beyond the convective core boundary at $r_{\mathrm{cc}}$. For stars with a well-developed core, the mixing in the convective core is instantaneous and the value of $\alpha_{\mathrm{mlt}}$ does not matter for the core region, but it does so for the thin convective envelope for stars with masses below some $2.5 \mathrm{M}_{\odot}$ (higher-mass stars have pure radiative envelopes). The calibration of $\alpha_{\text {mlt }}$ has been the subject of much research, with a commonly accepted calibration being $\alpha_{\text {mlt }}=1.8$ (Joyce \& Chaboyer 2018). As such, we fix $\alpha_{\text {mlt }}$ to this value.

An isochrone $\phi_{i, j}$ at a given age $\tau_{j}$ is then a single function in the parameter space, with each effective temperature corresponding to a single surface gravity, or luminosity. A comparison of isochrones $\phi_{i, j}$ and $\phi_{i+n, j}$ with a factor eight increase in CBM efficiency can be seen by comparing the orange $\left(\phi_{i, j}\right)$ and blue $\left(\phi_{i+n, j}\right)$ isochrones in the panels of Fig. 3 .

An isochrone-cloud is then the region in the HRD or CMD at a given age, $\tau_{j}$, covered by all of the isochrones $\phi_{j}=\sum_{i} \phi_{i, j}$ spanning the range of internal mixing values, but which still have a single metallicity. In practice an isochrone-cloud is then just the collection of the isochrones $\phi_{j}$. Examples of isochroneclouds are seen in grey in the panels of Fig. 3, where we note that a given isochrone-cloud is always bound by the isochrones with the most (orange isochrone) and least (blue isochrone) amount of interior mixing considered. Following the panels of Fig. 3, we see how the TAMS gradually expands in both colour and magnitude as the age of the cloud increases, naturally producing the same morphology as is observed in the eMSTO. This spreading is driven by the spread in core masses of the stars in the population with different amounts of mixing, and hence different levels of enhancement. An example of the evolution of the fractional core mass distribution for each of the isochrone-clouds shown in Fig. 3 is seen in comparing the distributions in Fig. 4. We notice that as the population ages, the fractional core mass distribution shifts to lower values and broadens.

As stated, we implement CBM as diffusive exponential overshooting in the definition by Paxton et al. (2011), to which we refer for details. We adopt the range of overshooting values calibrated by asteroseismic studies as: $f_{\mathrm{CBM}} \in 0.005-0.040$. We implement REM as a scaled profile described by Pedersen et al. (2018) and based on Rogers \& McElwaine (2017) with 

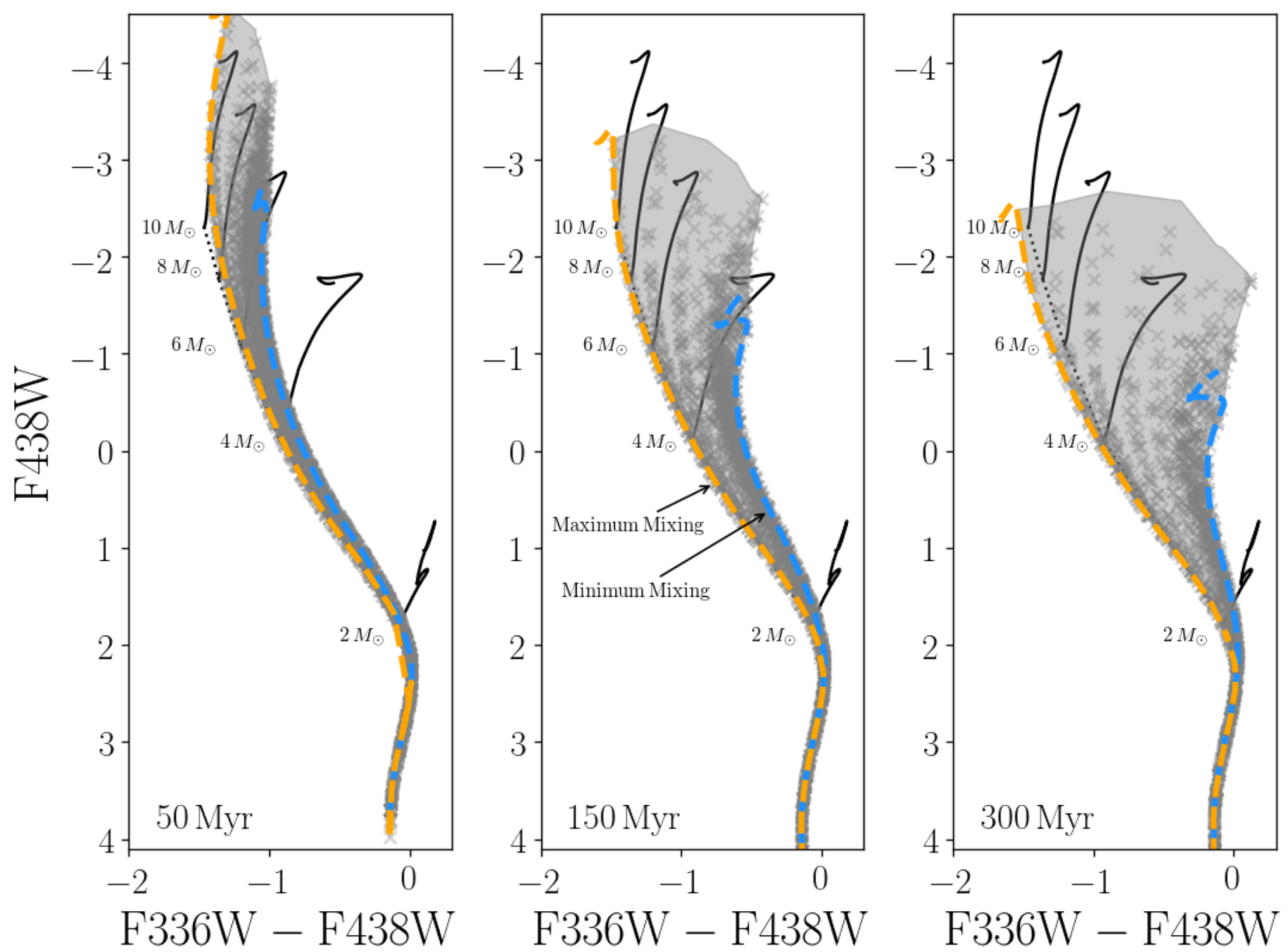

Fig. 3. Example isochrone-clouds (grey-region) as a function of age. Isochrone-clouds shown at $35 \mathrm{Myr}$ (left), $100 \mathrm{Myr}$ (middle), and $150 \mathrm{Myr}$ $($ right), with individual isochrones plotted as grey point, and evolutionary tracks over-plotted in black. The masses for the evolutionary tracks are denoted at the ZAMS for each track. The blue dashed line denotes the isochrone with the minimum amount of interior mixing and the orange dashed line represents the isochrone with the maximum amount of interior mixing in our model grid.

the transitional mixing value being: $\log \left(D_{\text {REM }}\right) \in 0-4$, corresponding with a mixing efficiency between 1 and $10000 \mathrm{~cm}^{2} \mathrm{~s}^{-1}$ at the bottom of the radiative envelope. As said, such levels have been assessed by $g$-modes in Kepler space photometry of core-hydrogen burning stars with a convective core, covering the ZAMS to the TAMS. $D_{\text {mix }}(r)$ profiles calibrated by $g$-modes are not yet available for hydrogen-shell burning stars. Space photometry that might be suitable to derive them is currently being gathered by TESS (Bowman et al. 2019b). As such, these profiles are fine to fit the eMSTO but need to get calibrated for post core-hydrogen burning phases for future fitting of the entire CMDs of clusters, including blue supergiants, rather than just the MS as we do here.

Our grid of isochrone-clouds covers birth masses, $M_{\star} \in$ $[1.2,25.0] M_{\odot}$, and three metallicities $Z=0.006,0.010,0.014$, along with the ranges of $D_{\mathrm{CBM}} \in[0.005,0.040]$ and $\log D_{\mathrm{REM}} \in$ $[0,4]$ derived from asteroseismic modelling of gravity-mode pulsators observed by the Kepler mission. This grid covers stellar models with convective core masses in the range $M_{\mathrm{cc}} / M_{\star} \in$ $[0,35] \%$, where a value of typically $\sim 10 \%$ occurs for ZAMS models that have no CBM or REM. As said, we limit the range in levels and shapes of mixing to those derived from asteroseismic modelling of galactic field single and binary stars of intermediate and high mass, mainly but not exclusively from Kepler space photometry - see MfA.

\section{Application to NGC 1850 and NGC 884}

\subsection{Fitting procedure}

Our isochrone-clouds consist of absolute magnitudes and bolometric corrections in all HST and Johnson filters. We choose to convert these to apparent magnitude, given a distance and extinction correction, rather than alter the data. As such, we take distance estimates to the two clusters derived from distance modulus and extinction estimates in the literature, yielding $d=42658$ pc for NGC 1850 (Bastian et al. 2016; Correnti et al. 2017; Yang \& Tian 2017; Yang et al. 2018) and $d=2250 \mathrm{pc}$ for NGC 884 (Kharchenko et al. 2013; Li et al. 2019b; Beasor et al. 2019). We calculate extinction coefficients for each filter using the York Extinction Solver (McCall 2004), assuming $R_{v}=3.1$.

Since we are concerned with fitting the eMSTO specifically for stars with a convective core, and not the general morphology of the cluster in the whole CMD, we make a cut in magnitude and colour for both clusters. For NGC 1850 we cut at $m_{F 439 W}>19$ and $m_{F 336 W}-m_{F 439 W}<0$, in accordance with 


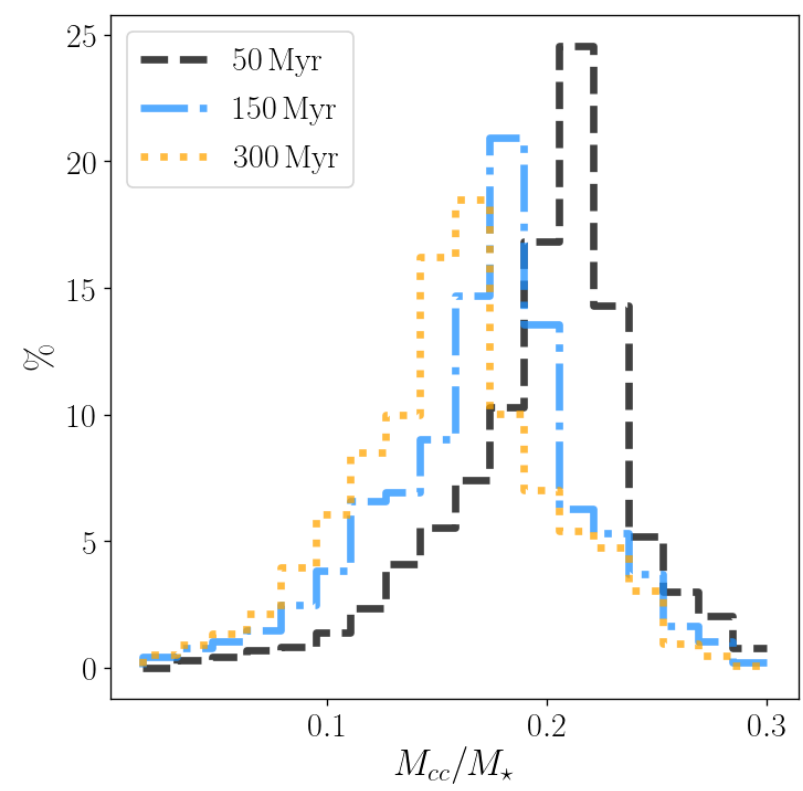

Fig. 4. Distribution of fractional core masses for three isochrone-clouds shown in panels of Fig. 3. The dashed black distribution represents the fractional core masses for the $50 \mathrm{Myr}$ isochrone-cloud, the blue dasheddotted distribution is for the $150 \mathrm{Myr}$ isochrone-cloud, and the orange dotted distribution is for the $300 \mathrm{Myr}$ isochrone-cloud.

Bastian et al. (2016), and for NGC 884 we cut at $G_{\mathrm{RP}}<14$ and $G_{\mathrm{BP}}-G_{\mathrm{RP}}<0.75$ following and using the Gaia DR2 data from Li et al. (2019b).

We fitted isochrone-clouds to the selected part of the CMD of each cluster, assuming that their members belong to one coeval population of single stars, at every age between 5 and $500 \mathrm{Myr}$ (in steps of $2.5 \mathrm{Myr}$ between 5 and $50 \mathrm{Myr}$ and steps of $10 \mathrm{Myr}$ between 50 and $500 \mathrm{Myr}$ ). Taking into account that the two free parameters of $D_{\mathrm{CBM}}$ and $D_{\mathrm{REM}}$ are correlated with each other and with the (core) mass and age of the star, we use the Mahalanobis distance (MD) as a merit function, rather than a $\chi^{2}$ (we refer to Aerts et al. 2018, for a definition and thorough discussion of its advantage in estimation of correlated parameters). For every cluster member, we calculate the MD with respect to every point in the isochrone-cloud, and select the point with the smallest MD as the best fit for that cluster member. Then, the MD for each cluster member is summed for each isochrone-cloud, to arrive at a total fit quality. We take the isochrone-cloud with the lowest total MD as the best fitted isochrone-cloud for the cluster.

So far, no suitable high-precision space photometry is available to perform ensemble asteroseismic modelling of stars in young (Myr old) open clusters. In absence of such data, we used the ensemble of $D_{\mathrm{CBM}}$ and $D_{\mathrm{REM}}$ estimated from asteroseismology of isolated field stars in the Milky Way in MfA as being representative for the occurring $D_{\text {mix }}(r)$ profiles inside single stars, as discussed above. With the aim to check how much of the width of measured eMSTOs can be explained with a single coeval population, we applied isochrone-cloud eMSTO fitting to two young clusters of different metallicity, one of which hosts numerous pulsators for which ground-based multi-colour photometry is available.

\section{2. $N G C 1850$}

We use the HST, spectroscopic, and Strömgren data of the LMC cluster NGC 1850 from the studies by Bastian et al. (2016,

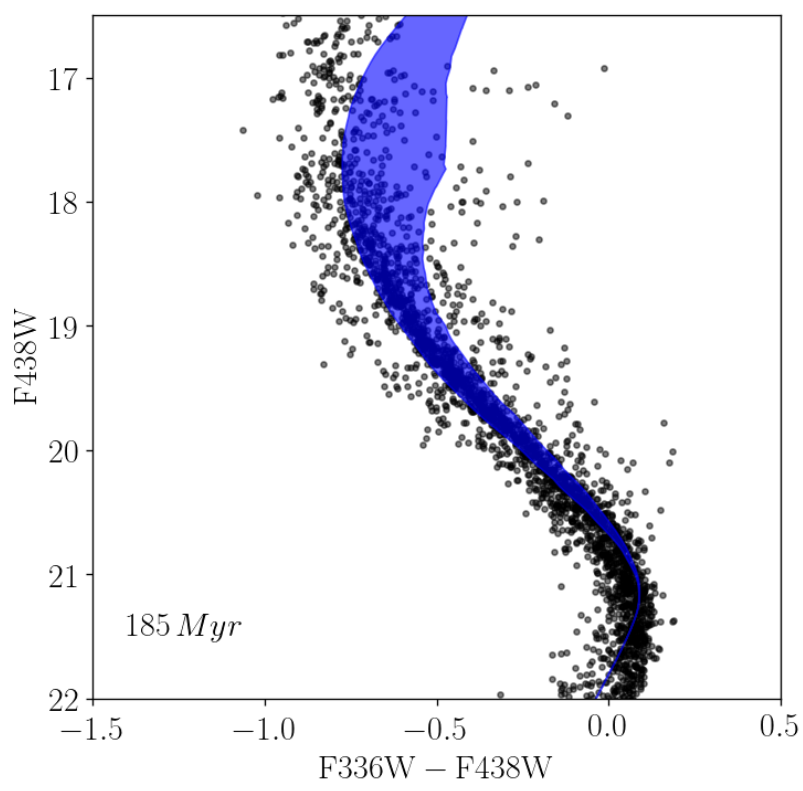

Fig. 5. Isochrone-cloud of co-eval population of single stars at $\sim 185$ Myr (blue) resulting from best eMSTO fitted for NGC 1850.

2017), Correnti et al. (2017), Piatti et al. (2019). These imply $Z=0.0061$, an eMSTO-based age of $\sim 80 \mathrm{Myr}$ and a turn-off mass between 4.6 and $5 M_{\odot}$ (Bastian et al. 2017). This places the eMSTO stars straight into the instability strip of the SPB stars. No suitable time series data is available to assess the variability of the cluster members. The bluest MS stars of the eMSTO of this LMC cluster have been interpreted in terms of slowly rotating single stars (D'Antona et al. 2017) or low-mass-ratio binaries (Yang et al. 2018). A major fraction of the eMSTO stars reveal $\mathrm{H} \alpha$ emission and are therefore interpreted as being fast rotators, but quantitative values of their $v \sin i$ are lacking so it is not possible to estimate at what fraction of the critical rate they rotate (Bastian et al. 2017).

We fitted each of the stars above the colour-magnitude cutoff with our isochrone-clouds at fixed $Z=0.006$, while letting the parameters for CBM and REM free in the intervals $D_{\mathrm{CBM}} \in$ $[0.005,0.040]$ and $\log D_{\text {REM }} \in[0,4]$. This results in a best fitted age estimate of $\sim 185 \mathrm{Myr}$, as illustrated in Fig. 5. As can be seen, the fit is quite appropriate for the eMSTO regime. The resulting age is $\sim 32 \%$ higher than the recent age estimation based on rotating Geneva models (see Bastian et al. 2017). The distributions of the (core) masses for the best isochrone-cloud fit are shown in Fig. 6. The masses cover the range $M \in[1.9,4.0] M_{\odot}$, which is lower than the turn-off mass reported by Bastian et al. (2017). This is due to the higher fractional core masses considered in our stellar models. The positions in the CMD are split up in the two leftmost panels of Fig. 6, which contain stars with $\mathrm{H} \alpha$ in absorption (leftmost panel) and stars with $\mathrm{H} \alpha$ in emission (middle panel), following this spectro-photometric information from Bastian et al. (2017). There is no obvious difference in position between these two populations of stars in the sense that they occur across the entire eMSTO, as already pointed out in Bastian et al. (2017). We note that while our isochrone-clouds explain a large portion of the morphology of the eMSTO, they still struggle to explain the apparent split MS, that is, the blue-most points. It has been suggested that this split-MS could be the product of a bi-modal rotation distribution (D'Antona et al. 2015), however, to date there have not been any $v \sin i$ measurements of those stars in this region to confirm or contrast this statement. 

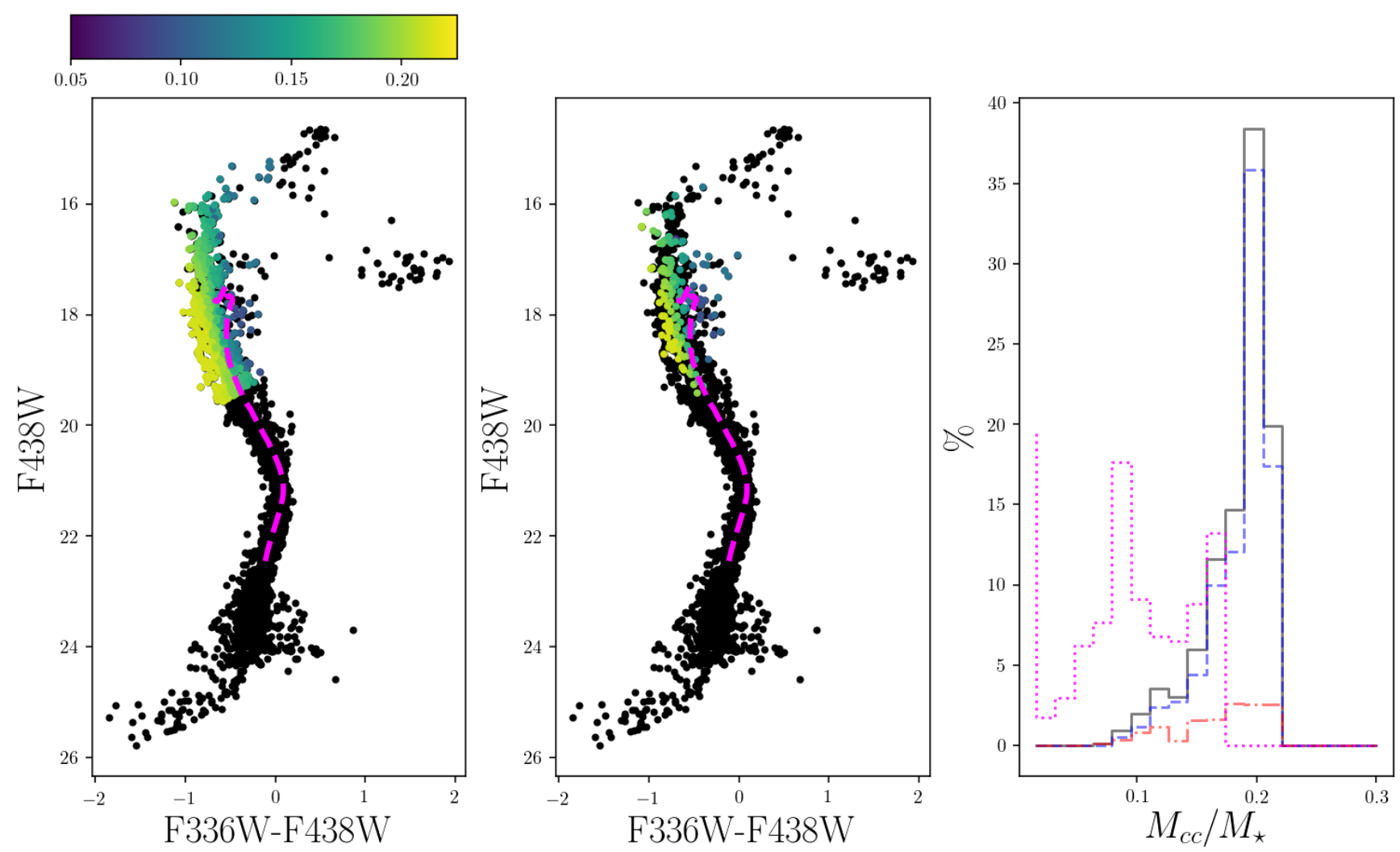

Fig. 6. Fitting results for NGC 1850 using isochrone-clouds with $Z=0.006$, leading to age of $\sim 185$ Myr. Left: observed stars in NGC 1850 without $\mathrm{H} \alpha$ emission, colour-coded according to the ratio of the convective core mass versus total stellar mass. Middle: observed stars in NGC 1850 with $\mathrm{H} \alpha$ emission colour-coded according to convective core mass. The isochrone with minimum amount of mixing at $185 \mathrm{Myr}$ is plotted in magenta for reference in both panels. Right: distribution of the fitted core mass as a fraction of total stellar mass, indicated in black for the full sample, in dashed blue for stars without $\mathrm{H} \alpha$ emission and in dashed-dotted red for stars with $\mathrm{H} \alpha$ emission. The distribution of fractional core mass for the $185 \mathrm{Myr}$ isochrone with minimum mixing is shown in dotted magenta.

Alternatively, we cannot rule out the possibility that a more sophisticated fitting methodology which optimises distance and extinction simultaneously with age could more accurately replicate the apparent split-MS instead.

The right panel of Fig. 6 shows the fractional core mass distribution (FCMD) for the total sample (black), the sample of stars with $\mathrm{H} \alpha$ emission (red), without $\mathrm{H} \alpha$ emission (blue), and the FCMD for those points along the isochrone with the minimum mixing in our grid (pink). The FCMD for NGC 1850 is a one-tailed skewed distribution ranging from 0.06 to 0.23 with a clear maximum at a fractional core mass of 0.2 and a sharp dropoff at higher values. The overall FCMD (black) is significantly shifted with respect to the one for the case of minimal mixing (pink-dotted), which represents models with standard core masses without CBM along the evolution. It can also be seen from the three panels that the distribution of $M_{\mathrm{cc}} / M_{\star}$ is uncorrelated with the $\mathrm{H} \alpha$ behaviour, while being cleanly segregated in the sense that stars with higher fraction of $M_{\mathrm{cc}} / M_{\star} \%$ (i.e. stars with a high amount of interior mixing) are situated on the blue side of the eMSTO. Our findings imply that the position in the eMSTO is connected with the relative ratio of the core versus overall stellar mass, and not with the surface rotation of the stars. This is not necessarily in contradiction with previous interpretations of stellar rotation being the dominant cause of the eMSTO, as our way of increased near-core and envelope mixing is not coupled to a specific physical process. Our findings show that efficient interior mixing, as it has been calibrated from asteroseismology for field stars, is sufficient to explain a large portion of the observed width of the eMSTO for non-rotating 1D evolutionary models. Our interpretation does not require stars to be rotating fast, and particularly not near critical, but rather suggests that an efficient mixing mechanism is at work in the near-core region of the star. The cause of this CBM could be rotation, or other phenomena such as waves, tides, etc.

\subsection{NGC 884}

NGC 884 is a bright northern galactic open clusters that has been observed in ground-based months-long time-series multi-colour photometry with the aim of detecting stellar oscillations in the cluster members (Saesen et al. 2010). This extensive monitoring was done in an attempt to perform cluster ensemble asteroseismology (Saesen et al. 2013). The campaign revealed 115 detected oscillation frequencies in 65 B-type stars in the cluster, covering amplitudes in the $V$ band between roughly 0.5 and $18 \mathrm{mmag}$ (Fig. 6 in Saesen et al. 2013). While those data are far less precise than Kepler and TESS space photometric time series and not suitable to perform asteroseismic modelling to derive $D_{\text {mix }}(r)$ for individual stars, this cluster ensemble of B-type pulsators did lead to an asteroseismic age range of $\sim 13.2-19.1 \mathrm{Myr}$, based on the detected pressure modes in eight $\beta$ Cep stars in the cluster. This result was obtained from stellar models with penetrative convection computed with the code CLÉS (Scuflaire et al. 2008).

The presence of numerous non-radial pulsators with a $v \sin i$ measurement in NGC 884 makes it one of the optimal clusters 


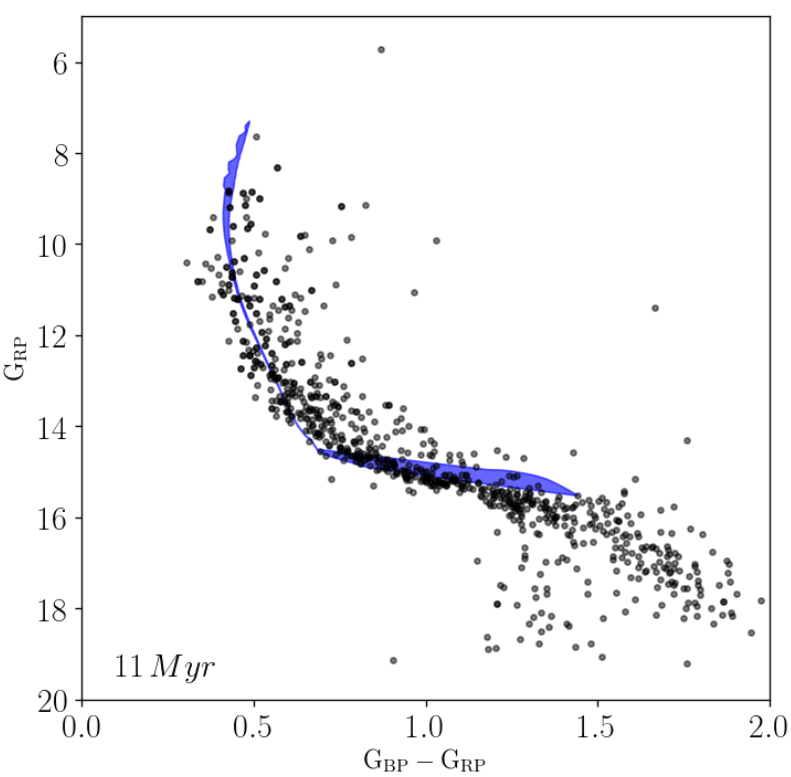

Fig. 7. Isochrone-cloud of co-eval population of single stars at $\sim 11 \mathrm{Myr}$ (blue) resulting from best eMSTO fitted for NGC 884.

that lends itself to test if its eMSTO bears any correlation with the oscillation properties of its members, rather than or in addition to their surface rotation. Moreover, in such a young cluster, only very efficient mixing processes that act on a timescale much shorter than the nuclear time scale will be able to build up a larger core mass due to CBM since the ZAMS. We test these hypotheses from isochrone-cloud fitting allowing for $\log D_{\text {REM }} \in$ $[0,4]$ as calibrated from MfA.

We fitted the observed eMSTO in the Gaia DR2 CMD used by $\mathrm{Li}$ et al. (2019b) with our isochrone-clouds, again assuming one coeval population of single stars. This time we fixed $Z=0.014$, given the occurrence of numerous multiperiodic B-type pulsators in the cluster. The excitation of stellar oscillations in such stars is based on the $\kappa$ mechanism. For this mechanism to work and excite pressure ( $p$-) modes in $\beta$ Cep stars, a $Z$ - (opacity) bump due to the iron-group elements must occur in the outer envelope of such stars, demanding $Z>0.010$ for the adopted solar mixture (Asplund et al. 2009) and OP opacity tables (Miglio et al. 2007).

Our isochrone-cloud fitting results in an age estimate of $\sim 11 \mathrm{Myr}$ and a stellar mass coverage of $M \in[1.7,17.8] M_{\odot}$, in agreement with Saesen et al. (2013) and with the fact that $p$ - and $g$-mode oscillations were detected in cluster members (Saesen et al. 2013). This age estimate is a slightly younger than the $14 \mathrm{Myr}$ estimate by Li et al. (2019b), who used the SYCLIST suite of Geneva rotating stellar models (Georgy et al. 2014). However, given the different model assumptions and fitting uncertainties, we consider these to results to be in agreement. Considering such model differences, our estimate is also in agreement with those of 13.2-19.1 Myr produced by Saesen et al. (2013), who ignored the effect of rotation on oscillation frequencies and made assumptions on mode identification. The result of our fitting are shown in Fig. 7 and is to be compared with Fig. 7 in Li et al. (2019b), who showed the case of a non-rotating population as well as one rotating at $90 \%$ critical.

The results for the estimates of the core masses are shown in the left panel of Fig. 8. The central panels of the figure show the stars in the cluster with measurements of $v \sin i$ (upper), and the frequency and amplitude of the dominant oscillation mode (middle and lower) as a function of the fractional core mass. We check all of these for statistically significant correlations via linear regression modelling, with the results listed in Table 1 . We find no significant correlations between either $v \sin i, f_{\mathrm{osc}}$, or $A_{\mathrm{osc}}$ and the fractional core masses.

The total FCMD (solid black distribution in the right panel of Fig. 8) ranges between 0.04 and 0.32 , with a clear maximum at a fractional core mass of 0.25 and an extended population of stars with higher fractional core masses. The fractional core mass distributions for those stars with $v$ sin $i$ estimates (red dashed-dotted distribution) and with $f_{\text {osc }}$ measurements (blue dashed distribution) are similar and show no clear trends. The resulting fitted distribution is different from the distribution of fractional core masses assuming a population with the least amount of interior mixing in the grid (pink-dotted distribution), indicating that also in this much younger cluster members, a significant amount of mixing occurs for its eMSTO stars. Pulsational wave mixing by IGW qualifies as an explanation, just as it provides a suitable mechanism for efficient AM transport (cf., Aerts et al. 2019; Bowman et al. 2019b, and references therein). Given that the stars at the eMSTO of NGC 884 are more massive than those for NGC 1850, and that the amplitude of detected IGW was found to be proportional with the stellar mass and luminosity and independent of metallicity (Bowman et al. 2019b), IGWs are predicted to be more efficient for more massive stars. This does not exclude other phenomena that could also act on the required short time scale to enlarge the core masses of the eMSTO stars.

\section{Discussion and conclusions}

NGC 1850 and NGC 884 are two young open clusters known to have significantly different age and metallicity. Their eMSTO is situated in the range of intermediate-mass B-type stars. From isochrone-cloud fitting, based on interior mixing levels as derived from asteroseismology of single field stars of similar mass, we find that the stars at the eMSTO of both clusters have enhanced core masses compared to standard models without core boundary and envelope mixing. As a result of the interior mixing, two stars with the same age and similar birth mass, but different interior mixing levels would appear in different locations on the CMD, where the star with enhanced mixing would be located blueward of its less mixed analogue.

From our isochrone-cloud fitting, we derive an age estimate of 11 Myr for NGC 884 and 185 Myr for NGC 1850 . We find that both of these estimates agree with previous estimates considering the differences in model physics and fitting methodologies. The relative core mass fractions reveal a broader distribution for the younger and more metal-rich cluster NGC 884 than for the older LMC cluster NGC 1850. This suggests that (a) CBM mechanism(s) are active on a time scale much shorter than the nuclear time scale and that the mechanism(s) are more effective as the stellar masses are higher.

Our isochrone-cloud modelling is based on sphericallysymmetric stellar models with enhanced core masses compared to standard 1D models without extra mixing. This enables us to explain the morphology of the eMSTO of the two clusters to a large extent. Just as with asteroseismic modelling of single and binary pulsators, the cause of the interior mixing was left open and could be due to rotation, pulsations, waves, tides, binary merging, etc. The larger the CBM, the higher the relative core mass fraction gets during the first part of the MS, so the bluer the stars occurs in the HRD compared to models without interior mixing. We do not find any relationship between the $v \sin i$ of the stars and the core mass fraction. 

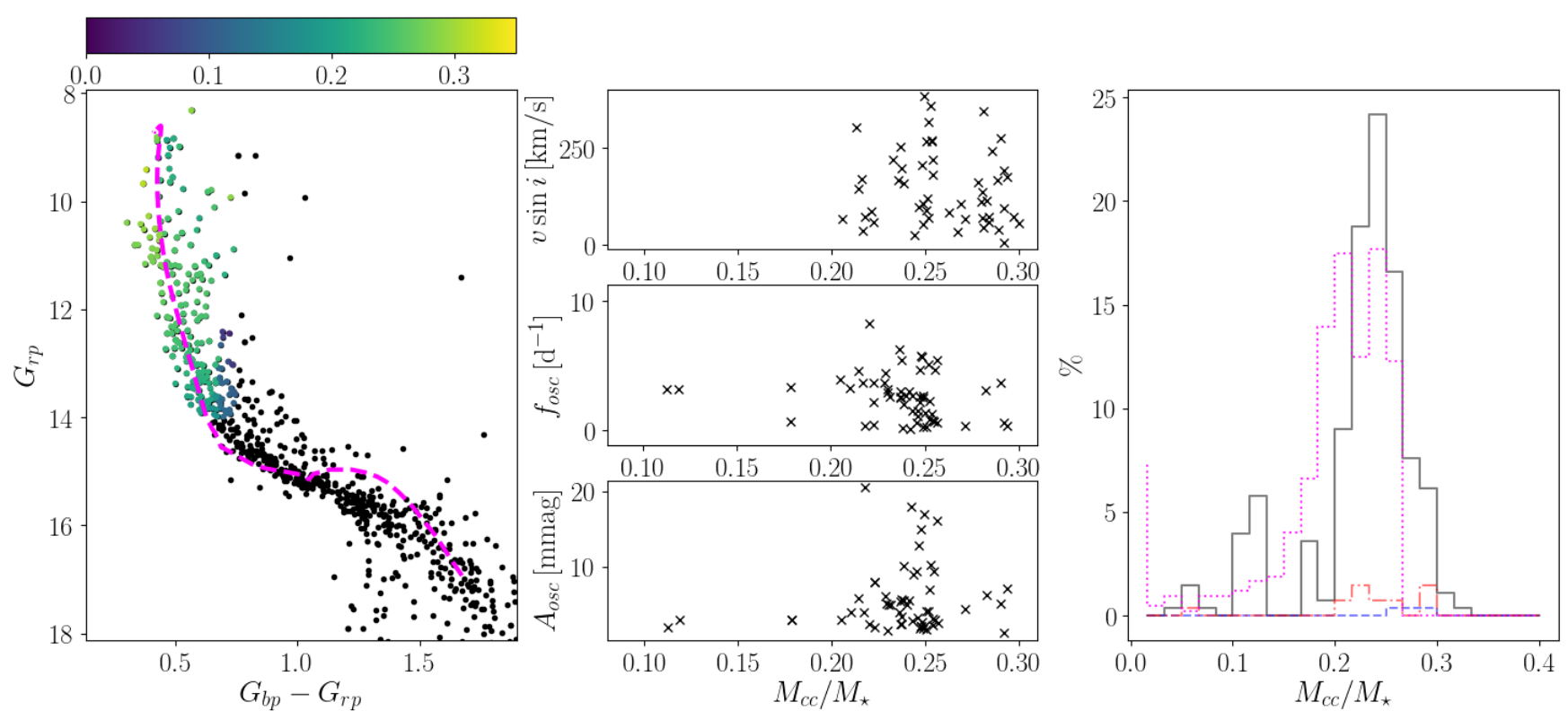

Fig. 8. Fitting results for NGC 884 using isochrone-clouds with $Z=0.014$, leading to age of $\sim 11$ Myr. Left: same as left panel of Fig. 6 . Upper, middle and lower panel of central plots: $v \sin i$, the dominant oscillation frequency and its amplitude vs. fractional core mass. Right: same as right panel of Fig. 6, but blue denotes stars with measured oscillations and red denotes stars with measured $v \sin i$. Pink dotted distribution is for those stars with the minimum amount of mixing.

Table 1. $R^{2}$ - and $p$-values of statistical regressions for various stellar parameters with the derived fractional core mass $M_{\mathrm{cc}} / M_{\star}$.

\begin{tabular}{lcc}
\hline \hline$y$-variable & $R^{2}$ & $p$ \\
\hline$v \sin i$ & 0.007 & 0.55 \\
$f_{\text {osc }}$ & 0.003 & 0.67 \\
$A_{\text {osc }}$ & 0.034 & 0.16 \\
\hline
\end{tabular}

With our work, we provide an entirely different explanation for the eMSTO than studies in the recent literature, which rely on models of stars with a spread of rotation rates, at least some of which are rotating near their critical velocity (as corroborated by the observation of $\mathrm{H} \alpha$ emission, with the assumption that such emission is caused by near critical rotation) such that colour $\left(T_{\text {eff }}\right)$ modifications due to geometric distortion and the Von Zeipel effect come into play (e.g. Georgy et al. 2019; Li et al. 2019b; Gossage et al. 2018). Rapid rotation and a high level of interior mixing are not mutually exclusive and both effects may be active in practice. However, it seems unrealistic to assume that almost all stars at the eMSTO rotate near critical. To that end, a sub-population of stars rotating at near critical rotation is still a candidate to explain the split-MS phenomenon. For NGC 884, high-precision spectroscopy revealed that the fastest rotating emission-line stars at the eMSTO rotate at about half their critical rate rather than near critical. For this cluster, the Von Zeipel effect hence does not offer a good explanation.

Our results indicate the need of higher core masses in stellar models for stars born with a mass above $\sim 1.4 M_{\odot}$. These higher core masses can be caused by enhanced amounts of interior mixing, which results in higher luminosities compared to the case where no extra mixing is considered in the near-core region. Mixing induced by the overall effect of penetrative convection, core overshooting, resonant standing gravity modes and/or dissipative IGW, is naturally expected for stars with masses higher than $1.4 M_{\odot}$, irrespective of their rotation rate. This cutoff mass has been found observationally from eMSTOs of numerous clusters (Goudfrooij et al. 2018). Rotational mixing due to meridional circulation and other instabilities induced by rotation (Heger et al. 2000; Aerts et al. 2019) would not necessarily lead to such a strict cutoff in mass. In order to explain this observed cutoff from rotating models, one can rely on magnetic braking induced by the outer convective envelope of stars (Georgy et al. 2019). Our mechanism, on the other hand, focuses on the innermost regions of the stars rather than on their envelope behaviour to explain the eMSTO and the cutoff at $1.4 M_{\odot}$ for the different eMSTO morphology in observed clusters.

We conclude for both considered clusters that isochroneclouds can reproduce to a large extent the morphology of their eMSTO in terms of interior mixing. Although we fitted only the eMSTO, the dimmer and redder regions of the CMD are adequately described by the best isochrone-cloud model, despite having had no influence on the fitting procedure. This suggests that our models are well calibrated. However, our tentative conclusions must be tested on a large sample of young open clusters covering a good spread in age, metallicity, and turn-off mass. Ideally, this work will be extended to incorporate space-based asteroseismology of YMCs to be delivered by TESS. We plan to take up such more systematic studies in future work.

Acknowledgements. We thank the referee for their comments which improved the manuscript. This work sparked from discussions among the authors during the 2018 Lorentz Workshop "Weighing Stars from Birth to Death: How to Determine Stellar Masses?"; we are grateful to the staff of the Lorentz Center for the kind hospitality and support. We are grateful to Chengyuan Li for having provided us with the Gaia CMD data from his paper on NGC 884 in electronic format. The research leading to these results has received funding from the European Research Council (ERC) under the European Union's Horizon 2020 research and innovation programme (grant agreements $\mathrm{N}^{\circ} 670519$ : MAMSIE with PI Aerts and $\mathrm{N}^{\circ} 646928$ : Multi-Pop with PI Bastian) and from the Research Foundation Flanders (FWO) under grant agreement G0A2917N: BlackGEM with PI Aerts. The computational resources and services used in this work were provided by the VSC (Flemish Supercomputer Center), funded by the Research Foundation - Flanders (FWO) and the Flemish Government - department EWI to PI Johnston. 


\section{References}

Aerts, C. 2015, in New Windows on Massive Stars, eds. G. Meynet, C. Georgy, J. Groh, \& P. Stee, IAU Symp., 307, 154

Aerts, C., Molenberghs, G., Kenward, M. G., \& Neiner, C. 2014, ApJ, 781, 88

Aerts, C., Van Reeth, T., \& Tkachenko, A. 2017, ApJ, 847, L7

Aerts, C., Molenberghs, G., Michielsen, M., et al. 2018, ApJS, 237, 15

Aerts, C., Mathis, S., \& Rogers, T. M. 2019, ARA\&A, 57, 35

Arnett, W. D., Meakin, C., Viallet, M., et al. 2015, ApJ, 809, 30

Asplund, M., Grevesse, N., Sauval, A. J., \& Scott, P. 2009, ARA\&A, 47, 481

Bastian, N., Niederhofer, F., Kozhurina-Platais, V., et al. 2016, MNRAS, 460, L20

Bastian, N., Cabrera-Ziri, I., Niederhofer, F., et al. 2017, MNRAS, 465, 4795

Bastian, N., Kamann, S., Cabrera-Ziri, I., et al. 2018, MNRAS, 480, 3739

Beasor, E. R., Davies, B., Smith, N., \& Bastian, N. 2019, MNRAS, 486, 266

Bodensteiner, J., Sana, H., Mahy, L., et al. 2019, A\&A, in press, https ://doi org $/ 10.1051 / 0004-6361 / 201936743$

Bouabid, M. P., Dupret, M. A., Salmon, S., et al. 2013, MNRAS, 429, 2500

Bowman, D. M., Aerts, C., Johnston, C., et al. 2019a, A\&A, 621, A135

Bowman, D. M., Burssens, S., Pedersen, M. G., et al. 2019b, Nat. Astron., 3, 760

Briquet, M., Morel, T., Thoul, A., et al. 2007, MNRAS, 381, 1482

Brott, I., de Mink, S. E., Cantiello, M., et al. 2011, A\&A, 530, A115

Buysschaert, B., Aerts, C., Bowman, D. M., et al. 2018, A\&A, 616, A148

Chaboyer, B., Demarque, P., \& Pinsonneault, M. H. 1995, ApJ, 441, 876

Claret, A. 2007, A\&A, 475, 1019

Claret, A., \& Torres, G. 2019, ApJ, 876, 134

Constantino, T., \& Baraffe, I. 2018, A\&A, 618, A177

Cordoni, G., Milone, A. P., Marino, A. F., et al. 2018, ApJ, 869, 139

Correnti, M., Goudfrooij, P., Bellini, A., Kalirai, J. S., \& Puzia, T. H. 2017, MNRAS, 467, 3628

D’Antona, F., Di Criscienzo, M., Decressin, T., et al. 2015, MNRAS, 453, 2637

D’Antona, F., Milone, A. P., Tailo, M., et al. 2017, Nat. Astron., 1, 0186

de Mink, S. E., Cantiello, M., Langer, N., et al. 2009, A\&A, 497, 243

Degroote, P., Aerts, C., Baglin, A., et al. 2010, Nature, 464, 259

Dotter, A. 2016, ApJS, 222, 8

Dotter, A., Conroy, C., Cargile, P., \& Asplund, M. 2017, ApJ, 840, 99

Dupree, A. K., Dotter, A., Johnson, C. I., et al. 2017, ApJ, 846, L1

Dupret, M. A., Grigahcène, A., Garrido, R., Gabriel, M., \& Scuflaire, R. 2005, A\&A, 435, 927

Ekström, S., Georgy, C., Eggenberger, P., et al. 2012, A\&A, 537, A146

Freytag, B., Ludwig, H. G., \& Steffen, M. 1996, A\&A, 313, 497

Fuller, J., Piro, A. L., \& Jermyn, A. S. 2019, MNRAS, 485, 3661

Gagnier, D., Rieutord, M., Charbonnel, C., Putigny, B., \& Espinosa Lara, F. 2019, A\&A, 625, A88

Georgy, C., Granada, A., Ekström, S., et al. 2014, A\&A, 566, A21

Georgy, C., Charbonnel, C., Amard, L., et al. 2019, A\&A, 622, A66

Gossage, S., Conroy, C., Dotter, A., et al. 2018, ApJ, 863, 67

Goudfrooij, P., Girardi, L., \& Correnti, M. 2017, ApJ, 846, 22

Goudfrooij, P., Girardi, L., Bellini, A., et al. 2018, ApJ, 864, L3

Heger, A., Langer, N., \& Woosley, S. E. 2000, ApJ, 528, 368

Hendriks, L., \& Aerts, C. 2019, PASP, 131, 108001

Higl, J., \& Weiss, A. 2017, A\&A, 608, A62

Johnston, C., Tkachenko, A., Aerts, C., et al. 2019a, MNRAS, 482, 1231

Johnston, C., Pavlovski, K., \& Tkachenko, A. 2019b, A\&A, 628, A25

Joyce, M., \& Chaboyer, B. 2018, ApJ, 856, 10

Kallinger, T., Weiss, W. W., Beck, P. G., et al. 2017, A\&A, 603, A13

Kamann, S., Bastian, N., Husser, T. O., et al. 2018, MNRAS, 480, 1689

Kharchenko, N. V., Piskunov, A. E., Schilbach, E., Röser, S., \& Scholz, R. D. 2013, A\&A, 558, A53

Kurtz, D. W., Saio, H., Takata, M., et al. 2014, MNRAS, 444, 102
Lagarde, N., Decressin, T., Charbonnel, C., et al. 2012, A\&A, 543, A108

Li, G., Van Reeth, T., Bedding, T. R., Murphy, S. J., \& Antoci, V. 2019a, MNRAS, 487, 782

Li, C., Sun, W., de Grijs, R., et al. 2019b, ApJ, 876, 65

Mackey, A. D., Broby Nielsen, P., Ferguson, A. M. N., \& Richardson, J. C. 2008, ApJ, 681, L17

Marino, A. F., Milone, A. P., Casagrande, L., et al. 2018a, ApJ, 863, L33

Marino, A. F., Przybilla, N., Milone, A. P., et al. 2018b, AJ, 156, 116

Marques, J. P., Goupil, M. J., Lebreton, Y., et al. 2013, A\&A, 549, A74

McCall, M. L. 2004, AJ, 128, 2144

Michaud, G., Alecian, G., \& Richer, J. 2015, in Atomic Diffusion in Stars (Heidelberg: Springer-Verlag), Astron. Astrophys. Lib.

Michielsen, M., Pedersen, M. G., Augustson, K. C., Mathis, S., \& Aerts, C. 2019, A\&A, 628, A76

Miglio, A., Montalbán, J., \& Dupret, M.-A. 2007, MNRAS, 375, L21

Milone, A. P., Marino, A. F., Di Criscienzo, M., et al. 2018, MNRAS, 477, 2640

Mombarg, J. S. G., Van Reeth, T., Pedersen, M. G., et al. 2019, MNRAS, 485, 3248

Moravveji, E., Aerts, C., Pápics, P. I., Triana, S. A., \& Vandoren, B. 2015, A\&A, 580, A27

Moravveji, E., Townsend, R. H. D., Aerts, C., \& Mathis, S. 2016, ApJ, 823, 130

Murphy, S. J., Fossati, L., Bedding, T. R., et al. 2016, MNRAS, 459, 1201

Niederhofer, F., Georgy, C., Bastian, N., \& Ekström, S. 2015, MNRAS, 453, 2070

Pápics, P. I., Briquet, M., Baglin, A., et al. 2012, A\&A, 542, A55

Pápics, P. I., Tkachenko, A., Van Reeth, T., et al. 2017, A\&A, 598, A74

Paxton, B., Bildsten, L., Dotter, A., et al. 2011, ApJS, 192, 3

Paxton, B., Cantiello, M., Arras, P., et al. 2013, ApJS, 208, 4

Paxton, B., Marchant, P., Schwab, J., et al. 2015, ApJS, 220, 15

Paxton, B., Schwab, J., Bauer, E. B., et al. 2018, ApJS, 234, 34

Paxton, B., Smolec, R., Schwab, J., et al. 2019, ApJS, 243, 10

Pedersen, M. G., Aerts, C., Pápics, P. I., \& Rogers, T. M. 2018, A\&A, 614, A128

Piatti, A. E., Pietrzyński, G., Narloch, W., Górski, M., \& Graczyk, D. 2019, MNRAS, 483, 4766

Ribas, I., Jordi, C., \& Giménez, Á. 2000, MNRAS, 318, L55

Rogers, T. M. 2015, ApJ, 815, L30

Rogers, T. M., \& McElwaine, J. N. 2017, ApJ, 848, L1

Rogers, T. M., Lin, D. N. C., McElwaine, J. N., \& Lau, H. H. B. 2013, ApJ, 772, 21

Saesen, S., Carrier, F., Pigulski, A., et al. 2010, A\&A, 515, A16

Saesen, S., Briquet, M., Aerts, C., Miglio, A., \& Carrier, F. 2013, AJ, 146, 102

Saio, H., Kurtz, D. W., Takata, M., et al. 2015, MNRAS, 447, 3264

Salaris, M., \& Cassisi, S. 2017, R. Soc. Open Sci., 4, 170192

Schmid, V. S., \& Aerts, C. 2016, A\&A, 592, A116

Scuflaire, R., Théado, S., Montalbán, J., et al. 2008, Ap\&SS, 316, 83

Seaton, M. J. 2005, MNRAS, 362, L1

Song, H. F., Maeder, A., Meynet, G., et al. 2013, A\&A, 556, A100

Szewczuk, W., \& Daszyńska-Daszkiewicz, J. 2017, MNRAS, 469, 13

Szewczuk, W., \& Daszyńska-Daszkiewicz, J. 2018, MNRAS, 478, 2243

Talon, S., Zahn, J. P., Maeder, A., \& Meynet, G. 1997, A\&A, 322, 209

Thoul, A. A., Bahcall, J. N., \& Loeb, A. 1994, ApJ, 421, 828

Tkachenko, A., Degroote, P., Aerts, C., et al. 2014, MNRAS, 438, 3093

Townsend, R. H. D., Goldstein, J., \& Zweibel, E. G. 2018, MNRAS, 475, 879

Van Reeth, T., Tkachenko, A., Aerts, C., et al. 2015, ApJS, 218, 27

Van Reeth, T., Tkachenko, A., \& Aerts, C. 2016, A\&A, 593, A120

Van Reeth, T., Mombarg, J. S. G., Mathis, S., et al. 2018, A\&A, 618, A24

Viallet, M., Meakin, C., Prat, V., \& Arnett, D. 2015, A\&A, 580, A61

Yang, W., \& Tian, Z. 2017, ApJ, 836, 102

Yang, Y., Li, C., Deng, L., de Grijs, R., \& Milone, A. P. 2018, ApJ, 859, 98 